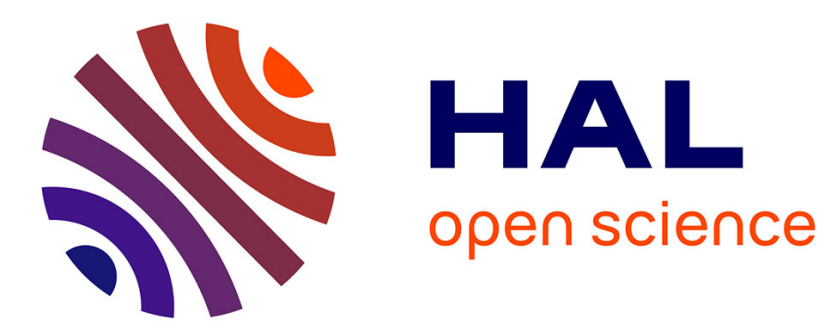

\title{
Adaptive Observer and Kalman Filtering
}

Khadidja Benzemrane, Gilney Damm, Giovanni L. Santosuosso

\section{To cite this version:}

Khadidja Benzemrane, Gilney Damm, Giovanni L. Santosuosso. Adaptive Observer and Kalman Filtering. 17th World Congress, International Federation of Automatic Control, IFAC, Jul 2008, Seoul, South Korea. pp.3865-3870, 10.3182/20080706-5-KR-1001.3675 . hal-00342773

\section{HAL Id: hal-00342773 https://hal.science/hal-00342773}

Submitted on 2 Apr 2014

HAL is a multi-disciplinary open access archive for the deposit and dissemination of scientific research documents, whether they are published or not. The documents may come from teaching and research institutions in France or abroad, or from public or private research centers.
L'archive ouverte pluridisciplinaire HAL, est destinée au dépôt et à la diffusion de documents scientifiques de niveau recherche, publiés ou non, émanant des établissements d'enseignement et de recherche français ou étrangers, des laboratoires publics ou privés. 


\title{
Adaptive Observer and Kalman Filtering
}

\author{
Khadidja Benzemrane* Gilney Damm** \\ Giovanni L. Santosuosso*** \\ * IBISC - CNRS/Université d'Evry Val d'Essonne, Evry, France \\ (e-mail: khadidja.benzemrane@ibisc.fr) \\ ** IBISC - CNRS/Université d'Evry Val d'Essonne, Evry, France \\ (e-mail: gilney.damm@ibisc.fr) \\ *** Dipartimento di Ingegneria Elettronica, Universitá di Roma Tor \\ Vergata, Via del Politecnico 1, Rome, Italy (e-mail: \\ santosuosso@ing.uniroma2.it)
}

\begin{abstract}
In this paper the problem of the speed estimation of an Unmanned Aerial Vehicle is addressed, when only the standard outputs (acceleration, angles and angular speeds) are available for measurement. We focus our analysis on a prototype drone - a 4 rotors helicopter robot- which is not equipped with GPS related devices and relies on the Inertial Measurement Unit (IMU) only. Two different approaches have been compared. The first one uses a classical method based on Kalman Filtering while the second solution is provided in the frame of adaptive observation theory. These estimators have been tested in two situations : when exact measurements are available and in the more realistic case of noisy acceleration measurements.
\end{abstract}

\section{INTRODUCTION}

The problem of speed estimation plays an important role in the context of vehicles control. For land moving robots, the odometer time derivative has a satisfactory performance while for large flying airships (manned or unmanned) velocity estimations can be obtained via approximate derivation of the successive measurements from GPS sensors, motivated by the small resulting errors compared to the measured variables. For fast aircrafts the standard procedure is integrating the acceleration and coupling this result to the derivative of GPS measurements.

Two critical issues arise in an "open loop" strategy like direct acceleration integration: an unknown constant estimation error is produced even when exact acceleration measurements are available while a random drift is induced by noisy acceleration estimations. In practice, numerical integration along with measurement noise induces a very fast growing velocity measurement error. Thus the information obtained from the GPS is used to bound this error in the framework of sensor fusion technique where initial conditions for acceleration integration are provided by GPS devices. This technique provides bounded errors that are related to the GPS order of precision. The resulting errors are usually small compared to the size of airships and the distance with respect to obstacles. In the same way, the control systems usually applied on these airships are robust enough to accept the residual disturbance on the speed estimation provided by these methods.

Unfortunately, the above estimation approach cannot be implemented on small drones less than 1 meter wide and flying at low speed that we consider in this note. This issue is even more relevant in indoors or simply urban applications. As a matter of fact, errors induced by a GPS system may reach many meters; a technical solution would be the use of a D-GPS system. These systems are known as centimetric GPS and have a precision of some centimetres, but they also are very expensive and low-range operating equipment. Another scheme is to use Doppler measurements coupled with GPS, that can actually increase velocity estimation accuracy.

In this work we focus our attention on the case when no GPS is available while Inertial Measurement Units (IMU) can be used. IMU actually represent a feasible choice: they are easily available, low cost devices. More important, they have a low size, limited weight, and provide very good airship measurements of accelerations, angles and angular speeds. However, the information provided by these devices needs to be processed to yield reliable velocity estimates. The latter quantity is necessary for an efficient drone control design, since most literature about small flying robots control assumes that at least the speeds are available (see Castillo et al. [2004], Guenardt et al. [2005], Pounds et al [2002]). This is motivated by the fact that is hardly possible for a human pilot (inboard or in tele-operation) to regulate a drone by giving references and trajectories as inputs based on the sole acceleration measurement. Notice that in the general case, the reliable estimation of the speed vector is still an open problem. Recently, control system and robotics communities have regained interest for the development of observers applied to UAVs due to the important developments of embedded electronics and micro-controllers. This technological improvement has motivated the testing of more sophisticated algorithms implemented in real time. This recent interest may be illustrated by recent publications as Guenardt et al. [2007], Bonnabel et al. [2006], Zhao et al. [2005] and Vik et al. [2001].

Motivated by previous arguments, this note compares an observation strategy that solves the problem of the speed estimation of an Unmanned Aerial Vehicle, when the linear acceleration, the angles and the angular speeds are 
available for measurement with a more classical estimator based on Kalman filtering (see Astrom et al. [1997]). The considered observer is based on robust nonlinear adaptive techniques as Marino et al. [1995] and Damm et al. [2004]. This strategy yields a satisfactory performance in a number of similar cases and for this reason has been chosen as first method. The authors believe that a strongly nonlinear and fast system as a drone must be treated by nonlinear techniques, while the number of disturbances and noisy measurements, as well as possibly different and time varying equilibrium points indicate the use of robust and adaptive techniques. Other techniques as variable structure observers and other high gain schemes should be considered in latter works, though the presence of noisy measurements may be a problem for those strategies.

In the present work, we focus our analysis on a prototype drone - a 4 rotors helicopter robot shown in Fig. 1 - produced to operate in an urban environment at the IBISC Laboratory - CNRS, University of Evry, which is not equipped with GPS related devices and relies on the Inertial Measurement Unit only (see Pradel et al. [2007]). The observation strategy final goal is to provide reliable estimations in order to design the robot's stabilizing control. The simulation results obtained on the model of the real drone in the presence of noise allows us to compare performances and the robustness of the observers.

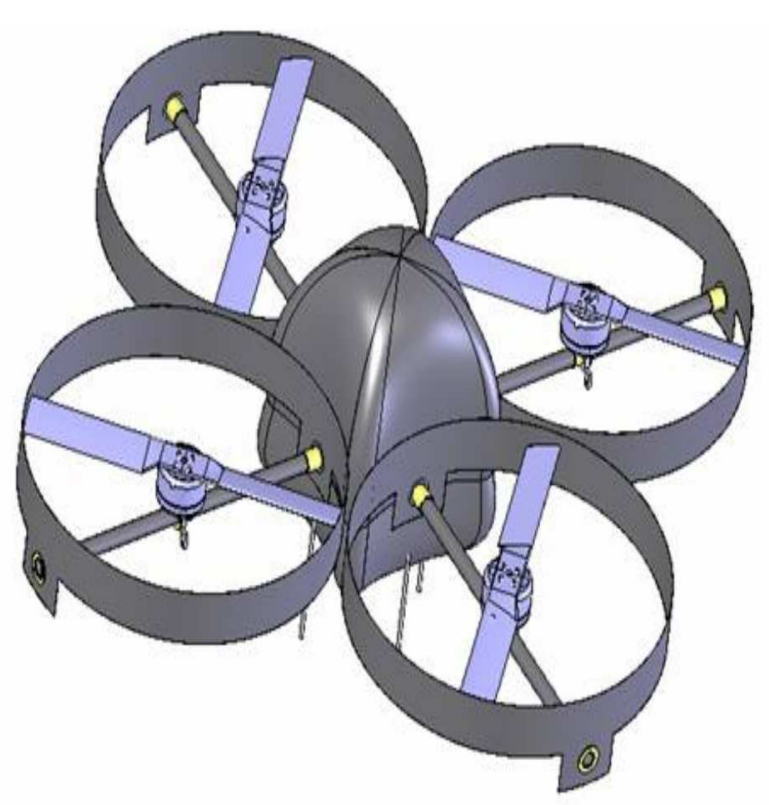

Fig. 1. Representation of the UAV

\section{PROBLEM FORMULATION}

The motion of a four rotor aerial robot can be described by the following equations (see Azouz et al. [2007]).

$$
\begin{aligned}
\dot{x}= & \cos \theta \cos \psi u \\
& +(\sin \phi \sin \theta \cos \psi-\cos \phi \sin \psi) v \\
& +(\cos \phi \sin \theta \cos \psi+\sin \phi \sin \psi) w \\
\dot{y}= & \cos \theta \sin \psi u \\
& +(\sin \phi \sin \theta \sin \psi+\cos \phi \cos \psi) v \\
& +(\cos \phi \sin \theta \sin \psi-\sin \phi \cos \psi) w \\
\dot{z}= & -\sin \theta u+\sin \phi \cos \theta v+\cos \phi \cos \theta w \\
\dot{\phi} & =p+(\sin (\phi) q+\cos (\phi) r) \tan (\theta) \\
\dot{\theta} & =\cos (\phi) q-\sin (\phi) r \\
\dot{\psi} & =(\sin (\phi) q+\cos (\phi) r) \cos (\theta)^{-1}
\end{aligned}
$$

$$
\begin{aligned}
I_{x x} \dot{p}= & -l_{b} k_{T}\left(\omega_{1}^{2} \cos \beta_{1}-\omega_{3}^{2} \cos \beta_{3}\right)-\left(I_{z z}-I_{y y}\right) r q \\
& -q I_{r}\left(\omega_{1} \cos \beta_{1}+\omega_{2}+\omega_{3} \cos \beta_{3}+\omega_{4}\right) \\
I_{y y} \dot{q}= & l_{b} k_{T}\left(\omega_{2}^{2}-\omega_{4}^{2}\right)-\left(I_{x x}-I_{z z}\right) r p \\
& -r I_{r}\left(\omega_{1} \sin \beta_{1}+\omega_{3} \sin \beta_{3}\right) \\
& +p I_{r}\left(\omega_{1} \cos \beta_{1}+\omega_{2}+\omega_{3} \cos \beta_{3}+\omega_{4}\right) \\
& +k_{M}\left(\omega_{3}^{2} \sin \beta_{3}-\omega_{1}^{2} \sin \beta_{1}\right)
\end{aligned}
$$$$
I_{z z} \dot{r}=-l_{b} k_{T}\left(\omega_{1}^{2} \sin \beta_{1}-\omega_{3}^{2} \sin \beta_{3}\right)-\left(I_{y y}-I_{x x}\right) p q
$$$$
+q I_{r}\left(\omega_{1} \sin \beta_{1}+\omega_{3} \sin \beta_{3}\right)
$$$$
+k_{M}\left(\omega_{3}^{2} \sin \beta_{3}+\omega_{4}^{2}-\omega_{1}^{2} \sin \beta_{1}-\omega_{2}^{2}\right)
$$$$
\begin{aligned}
\dot{u}= & (-q w+r v-g \sin \theta)-\frac{k_{T}}{m}\left(\omega_{1}^{2} \sin \beta_{1}+\omega_{3}^{2} \sin \beta_{3}\right) \\
\dot{v}= & (-r u+p w+g \sin \phi \cos \theta) \\
\dot{w}= & (-p v+q u+g \cos \phi \cos \theta) \\
& -\frac{k_{T}}{m}\left(\omega_{1}^{2} \cos \beta_{1}+\omega_{2}^{2}+\omega_{3}^{2} \cos \beta_{3}+\omega_{4}^{2}\right)
\end{aligned}
$$

where $\eta_{1} \triangleq\left[\begin{array}{lll}x & y & z\end{array}\right]^{T}$ is the position vector represented in the global reference frame, $\eta_{2} \triangleq\left[\begin{array}{lll}\phi & \theta & \psi\end{array}\right]^{T}$ is the Euler angles vector represented in the global reference frame (roll pitch and yaw respectively), $\nu_{1} \triangleq\left[\begin{array}{lll}u & v & w\end{array}\right]^{T}$ is the speed vector represented in the local reference frame (surge, sway and heave respectively) and $\nu_{2} \triangleq\left[\begin{array}{lll}p & q & r\end{array}\right]^{T}$ is the angular speed vector represented in the local reference frame. In this model, the control input vector is $\Omega=$ $\left[\begin{array}{llllll}\omega_{1} & \omega_{2} & \omega_{3} & \omega_{4} & \beta_{1} & \beta_{3}\end{array}\right]^{T}$ where $\omega_{i}, \quad i=1 \ldots 4$, are the angular speed of the 4 rotors, and $\beta_{1}$ and $\beta_{3}$ represent the orientation of the rotors 1 and 3 .

Constants $k_{T}$ and $k_{M}$, respectively, relate rotor speeds along with resulting thrust and torque; $l_{B}$ is the lenght of each drone's arm and $I_{r}$ is the rotor's inertia moment constant.

The paper task is to compare the performances of two different observers designed to provide estimation for the unmeasurable state variables $\nu_{1}$, based on the measurable variables $\eta_{2}, \dot{\nu}_{1}$ and $\nu_{2}$ given by the standard sensors embedded in the drone.

The two considered observers are respectively an adaptive observer designed to be robust with respect to measurement noise and a more classical estimation strategy based on Kalman filtering. 
The interest in introducing a tuning parameter of the adaptive observer, to enhance the quality of the estimation, will be illustrated by simulation results.

\section{ROBUST ADAPTIVE OBSERVER}

In this section, we describe the estimation strategy presented in Benzemrane et al. [2007], following the techniques presented in Marino et al. [2004], as well as an outline of its stability proof.

First, the system (4) can be re-writter as :

$$
\begin{aligned}
\dot{\nu}_{1}(t) & =A(t) \nu_{1}(t)+b(t) \\
y & =\dot{\nu}_{1}
\end{aligned}
$$

where $A, b$ and $y$ are defined as

$$
\begin{gathered}
A(t)=\left[\begin{array}{ccc}
0 & r & -q \\
-r & 0 & p \\
q & -p & 0
\end{array}\right] \\
b(t)=\left[\begin{array}{c}
-g \sin \theta-\frac{k_{T}}{m}\left(\omega_{1}^{2} \sin \beta_{1}+\omega_{3}^{2} \sin \beta_{3}\right) \\
g \sin \phi \cos \theta \\
g \cos \phi \cos \theta-\frac{k_{T}}{m}\left(\omega_{1}^{2} \cos \beta_{1}+\omega_{2}^{2}+\omega_{3}^{2} \cos \beta_{3}+\omega_{4}^{2}\right)
\end{array}\right] \\
y(t)=\dot{\nu_{1}}=\left[\begin{array}{c}
\dot{u} \\
\dot{v} \\
\dot{w}
\end{array}\right]
\end{gathered}
$$

In order to quantify the observer performance in case of measurement noise on the acceleration signals, we propose to take into account an additional measurement noise. We assume that a disturbed acceleration $a_{\mu}$ is available; $a_{\mu} \triangleq \dot{\nu}_{1}+\mu(t)$, where the vector $\mu \in R^{3}$ represents the measurement noise such that (defining $\mu_{M} \in R^{+}$):

$$
\|\mu\| \leq \mu_{M}
$$

Consider the cascaded filters with matrix states $M_{\mu} \in R^{3} \times$ $R^{3}, Q_{\mu} \in R^{3} \times R^{3}$, with arbitrary initial conditions (with $\left.\left|Q_{\mu}(0)\right|>0\right)$ defined as :

$$
\begin{gathered}
\dot{M}_{\mu}=-\bar{\alpha} M_{\mu}+A(t) \\
\dot{Q}_{\mu}=-\beta Q_{\mu}-\frac{k}{4} Q_{\mu} Q_{\mu}^{T} Q_{\mu}+M_{\mu}^{T} M_{\mu}
\end{gathered}
$$

where $\bar{\alpha} \in R^{+}$is such that $\bar{\alpha}=\alpha+\frac{k}{4}, \beta, k \in R^{+}$are tuning parameters chosen by the designer, along with the two vector systems with state $\rho_{\mu} \in R^{3}, \delta_{\mu} \in R^{3}$

$$
\begin{gathered}
\dot{\rho}_{\mu}=-\bar{\alpha} \rho_{\mu}+A(t) \nu_{1}+\mu+M_{\mu} a_{\mu} \\
=-\bar{\alpha} \rho_{\mu}+a_{\mu}-b(t)+M_{\mu} a_{\mu} \\
\dot{\delta_{\mu}}=-\beta \delta_{\mu}+\rho_{\mu} \\
\triangleq-\beta \delta_{\mu}+\tilde{z}_{\mu}+M_{\mu} \nu_{1}
\end{gathered}
$$

with arbitrary initial conditions.
In fact, by setting $\tilde{z}_{\mu}=\rho_{\mu}-M_{\mu} \nu_{1}$, we now have :

$$
\dot{\tilde{z}}_{\mu}=-\bar{\alpha} \tilde{z}_{\mu}+\left(M_{\mu}+I\right) \mu
$$

We introduce an estimate of the linear velocity

$$
\hat{\nu}_{1}=\left[\begin{array}{c}
\hat{u} \\
\hat{v} \\
\hat{w}
\end{array}\right]
$$

along with a filter vector state $\xi_{\mu} \in R^{3}$ that satisfy the differential equations :

$$
\begin{aligned}
\dot{\xi_{\mu}}= & -\beta \xi_{\mu}-\frac{k}{4} Q_{\mu} Q_{\mu}^{T} \xi+Q_{\mu} a_{\mu}-\dot{M}_{\mu}^{T} \delta_{\mu} \\
& -\frac{k}{4} Q_{\mu} Q_{\mu}^{T} M_{\mu}^{T} \delta_{\mu} \\
\dot{\hat{\nu}}_{1} & =\gamma\left(-Q_{\mu} \hat{\nu}_{1}+M_{\mu}^{T} \delta_{\mu}+\xi_{\mu}\right)+a_{\mu}
\end{aligned}
$$

with $\gamma \in R^{3}$ tuning parameter to be chosen by the designer By setting the estimation error variables $\tilde{\nu}_{1} \triangleq \nu_{1}-\hat{\nu}_{1}$ and $\chi_{\mu} \triangleq Q \nu_{1}-M_{\mu}^{T} \delta_{\mu}-\xi_{\mu}$, we obtain :

$$
\begin{gathered}
\dot{\chi}_{\mu}=-\left(\beta+\frac{k}{4} Q_{\mu} Q_{\mu}^{T}\right) \chi_{\mu}-M_{\mu}^{T} \tilde{z}_{\mu}-Q_{\mu} \mu \\
\dot{\tilde{\nu}}_{1}=\gamma\left(-Q_{\mu} \tilde{\nu}_{1}+\chi_{\mu}\right)-\mu
\end{gathered}
$$

From (14) along with (10) and (13), and if matrix Q is positive definite, we may guarantee the exponential convergence of estimation error. This result is based on the assumption:

Hypothesis 1. There are positive integers $T, K_{1}^{*}$ and $K_{2}^{*}$ such that

$$
K_{1}^{*} I \geq \int_{t}^{t+T} A(\tau)^{T} A(\tau) d \tau \geq K_{2}^{*} I \quad \text { for all } t \in \mathbf{R}
$$

Remark 1. Hypothesis 1 is a mathematical formulation of the properties that $\mathrm{A}(\mathrm{t})$ is bounded and persistently exciting (PE). The first is easily obtained for a physical system as the drone, while the second is obtained if the UAV tracks a trajectory which is in practice a small periodic orbit. Besides, airships dynamics provides vibrations in real applications, that may already give persistency of excitation.

Proposition 1. Consider the UAV model described by (1)-(4). Under Hypothesis 1 , if the variables $\eta_{2}, \nu_{2}, a_{\mu}$ are available for measurement, the dynamic observer described by (6), (7), (8), (9) with state $M_{\mu}(t) \in R^{3} \times R^{3}, Q_{\mu}(t) \in$ $R^{3} \times R^{3}, \rho_{\mu}(t) \in R^{3}, \delta_{\mu}(t) \in R^{3}, \hat{\nu}_{1}(t) \in R^{3}$, is such that for any given $\epsilon>0$, there exist a suitable combination of the tuning parameters yielding $\mathcal{L}_{2}$ and $\mathcal{L}_{\infty}$ gains from the measurement error $\mu$ to the estimations error $\left(\nu_{1}-\hat{\nu}_{1}\right)$ smaller than $\epsilon$, for any initial conditions $M_{\mu}(0) \in \mathrm{R}^{3} \times \mathrm{R}^{3}$, $Q_{\mu}(0) \in \mathrm{R}^{3} \times \mathrm{R}^{3}, \rho_{\mu}(0) \in \mathrm{R}^{3}, \delta_{\mu}(0) \in \mathrm{R}^{3}, \hat{\nu}_{1}(0) \in \mathrm{R}^{3}$ with $\left|Q_{\mu}(0)\right|>0$.

Proof 1. Stability can be shown by following standard Lyapunov arguments. 


$$
V=\frac{1}{2} \kappa_{1} \tilde{\nu}_{1}^{T} \tilde{\nu}_{1}+\frac{1}{2} \kappa_{2} \chi_{\mu}^{T} \chi_{\mu}+\frac{1}{2} \kappa_{3} \tilde{z}_{\mu}^{T} \tilde{z}_{\mu}
$$

By computing the time derivative of $V$, we have :

$$
\begin{aligned}
\dot{V} & =-\kappa_{1} \gamma \tilde{\nu}_{1}^{T} Q_{\mu} \tilde{\nu}_{1}-\kappa_{2} \beta \chi_{\mu}^{T} \chi_{\mu}-\kappa_{2} \frac{k}{4} \chi_{\mu}^{T} Q_{\mu} Q_{\mu}^{T} \chi_{\mu} \\
& -\kappa_{3}\left(\alpha+\frac{k}{4}\right) \tilde{z}_{\mu}^{T} \tilde{z}_{\mu}+\kappa_{1} \gamma \tilde{\nu}_{1}^{T} \chi_{\mu}-\kappa_{2} \chi_{\mu}^{T} M_{\mu}^{T} \tilde{z}_{\mu} \\
& -\kappa_{1} \tilde{\nu}_{1}^{T} \mu-\kappa_{2} \chi_{\mu}^{T} Q_{\mu} \mu+\kappa_{3} \tilde{z}_{\mu}^{T}\left(M_{\mu}+I\right) \mu
\end{aligned}
$$

As stated in Hypothesis 1, matrix $Q_{\mu}$ must be positive definite. Defining now the constants $\left|\lambda_{\max }\left(M_{\mu}\right)\right|=c_{1}$, $\lambda_{\min }\left(Q_{\mu}\right)=c_{2}$, we obtain :

$$
\begin{aligned}
\dot{V} \leq & -\kappa_{1} \gamma c_{2}\left\|\tilde{\nu}_{1}\right\|^{2}-\kappa_{2} \beta\left\|\chi_{\mu}\right\|^{2}-\kappa_{3} \alpha\left\|\tilde{z}_{\mu}\right\|^{2} \\
& +\kappa_{1} \gamma \frac{c_{2}}{2}\left\|\tilde{\nu}_{1}\right\|^{2}+\kappa_{1} k\left\|\tilde{\nu}_{1}\right\|^{2}+\frac{\kappa_{1} \gamma}{2 c_{2}}\left\|\chi_{\mu}\right\|^{2} \\
& +\frac{\kappa_{2} \beta}{4}\left\|\chi_{\mu}\right\|^{2}+\frac{c_{1}^{2} \kappa_{2}}{\beta}\left\|\tilde{z}_{\mu}\right\|^{2}+\frac{\kappa_{3}}{k}\left(c_{1}+1\right)^{2}\|\mu\|^{2} \\
& +\frac{\kappa_{2}}{k}\|\mu\|^{2}+\frac{\kappa_{1}}{k}\|\mu\|^{2}
\end{aligned}
$$

By choosing :

$$
\begin{aligned}
\kappa_{1} & =\frac{1}{\gamma} \\
\kappa_{2} & =\frac{2}{\beta c_{2}} \\
\kappa_{3} & =\frac{2 \kappa_{2} c_{1}^{2}}{\beta \alpha}=\frac{4 c_{1}^{2}}{\beta^{2} \alpha c_{2}}
\end{aligned}
$$

and by setting the design condition $\gamma>\frac{2 k}{c_{2}}$, we may finally state that for a suitable $\alpha_{i} \in \mathrm{R}^{+}$, equation (16) can be expressed as:

$$
\dot{V} \leq-\alpha_{1}\left\|\tilde{\nu}_{1}\right\|^{2}-\alpha_{2}\left\|\chi_{\mu}\right\|^{2}-\alpha_{3}\left\|\tilde{z}_{\mu}\right\|^{2}+\frac{\alpha_{4}}{k}\|\mu\|^{2}
$$

which guarantees arbitrary $\mathcal{L}_{\infty}$ and $\mathcal{L}_{2}$ robustness from the measurement error $\mu$ to the estimation errors $\tilde{\nu}_{1}, \chi_{\mu}$ and $\tilde{z}_{\mu}$.

Remark 2. An interesting issue arises from previous arguments. The problem of estimating linear velocities from accelerations is known as very difficult in terms of the existence of observability. As a matter of fact, the linearization of this system around the equilibrium point is non-observable.

The adaptive observer requires the classical persistence of excitation condition to obtain correct estimations. It is interesting to remark that to fulfil this condition automatically implies in the existence of observability of the linearized system. This is the reason that we may apply other observers like the Extended Kalman Filter (see Makhloufi [2007]) for this system since we use a trajectory that satisfies the same condition. The persistence of excitation condition reflects then the characteristics of observability of the system.

\section{SIMULATION RESULTS}

In this section we illustrate the behaviour of the presented observer designed to estimate the linear velocity of an UAV based on the measurable angles, angular velocities and linear accelerations. These simulations also compare it to an Extended Kalman Filter considered here as a benchmark.

In all simulations, the observer estimations have initial conditions set to zero, while the desired states are time varying (and different from zero at $t=0$ ). In all simulations, both observers track the desired time varying states, with different performances and noise rejections. We have used the following parameters values:

$$
\begin{array}{ll}
m=2.500 \mathrm{Kg} & k_{T}=10^{-5} \mathrm{N.s}^{2} \\
l_{b}=23 \mathrm{~cm} & I_{R}=10010^{-7} \mathrm{Kgm}^{2} \\
I_{x x}=22493110^{-7} \mathrm{Kgm}^{2} & I_{y y}=22261110^{-7} \mathrm{Kgm}^{2} \\
I_{z z}=32513010^{-7} \mathrm{Kgm}^{2} & k_{M}=910^{-5} \mathrm{Ns}^{2} \mathrm{~m} \\
u_{G}=0.032 \mathrm{~m} &
\end{array}
$$

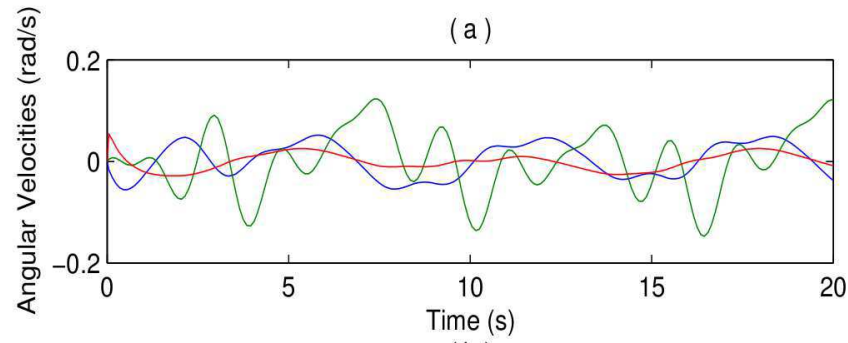

(b)

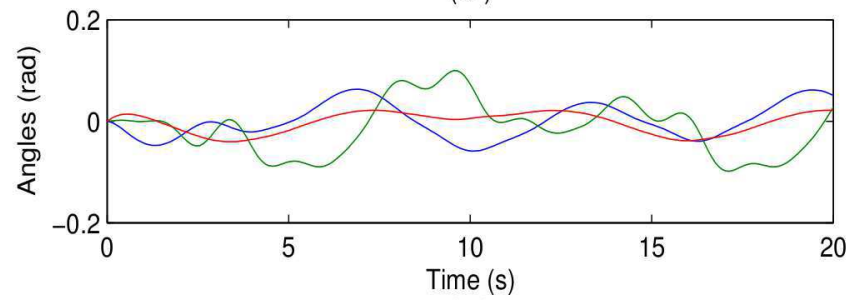

(c)

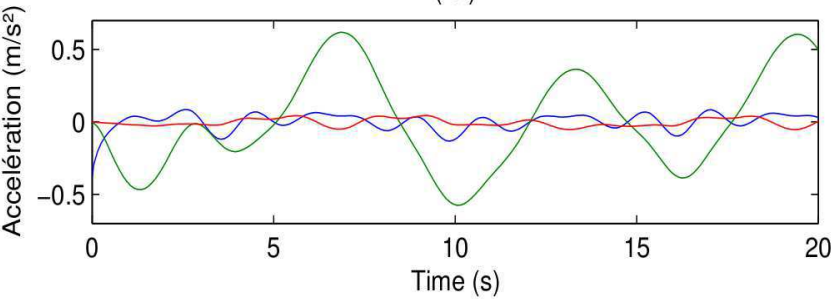

Fig. 2. (a)Angular Speed, (b)Angles, (c) Acceleration

In Fig. 2 the angular speeds, the angles, and the accelerations are plotted. As shown above, the persistency of excitation condition is needed to guarantee the observability of the system. Small periodic orbits were considered in order to satisfy the persistency of excitation condition.

We have then considered additive measurement noise. The measured acceleration is presented in Fig. 3, while Fig. 
4 shows the velocity that has to be estimated by the observer.

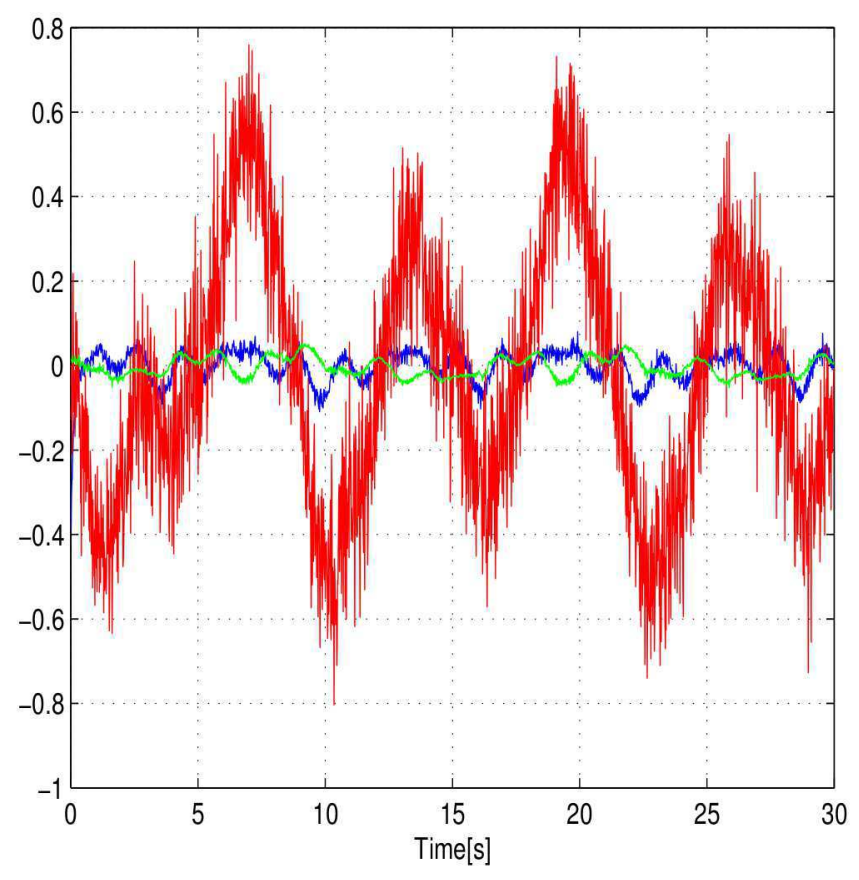

Fig. 3. Acceleration

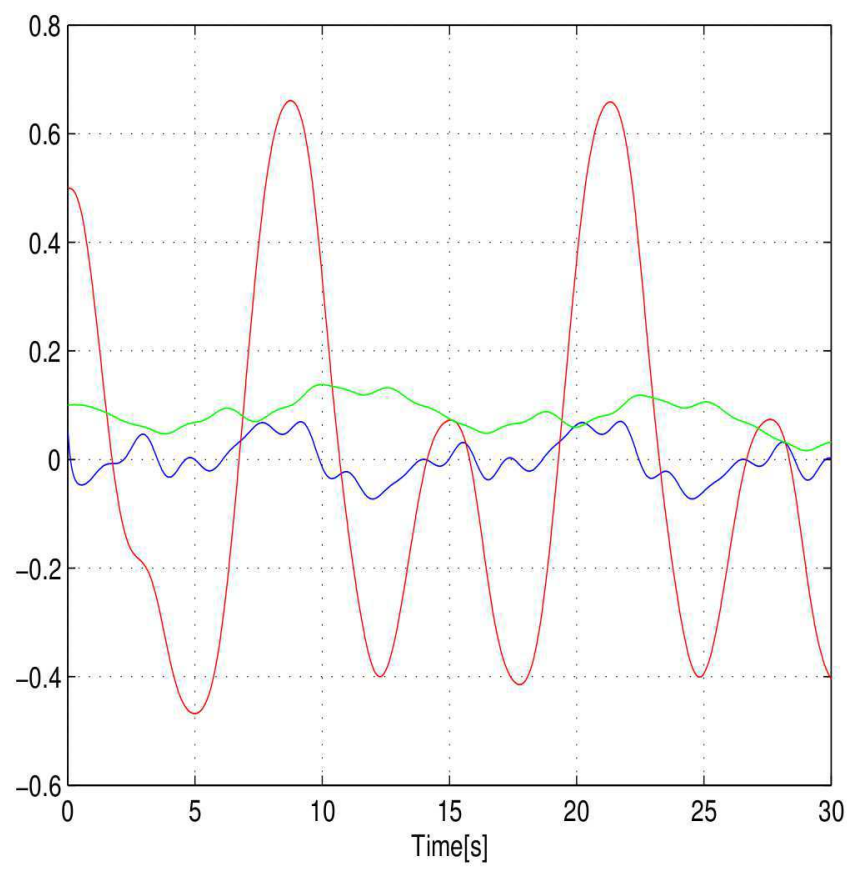

Fig. 4. Linear velocity to estimate

The first simulations are undertaken using the adaptiv observer, where the disturbance is attenuated followin the design parameter $k$. To describe the effect of th: parameter, we present two simulations using different values of $k$. In Fig. 5, we show the results of the simulations using $k=0$ and $k=50$. The main effects of larger values of $k$ are a greater attenuation, in trend of a slower convergence rate. These results are in accordance with our claims, and illustrate the mechanisms of the tuning procedure.
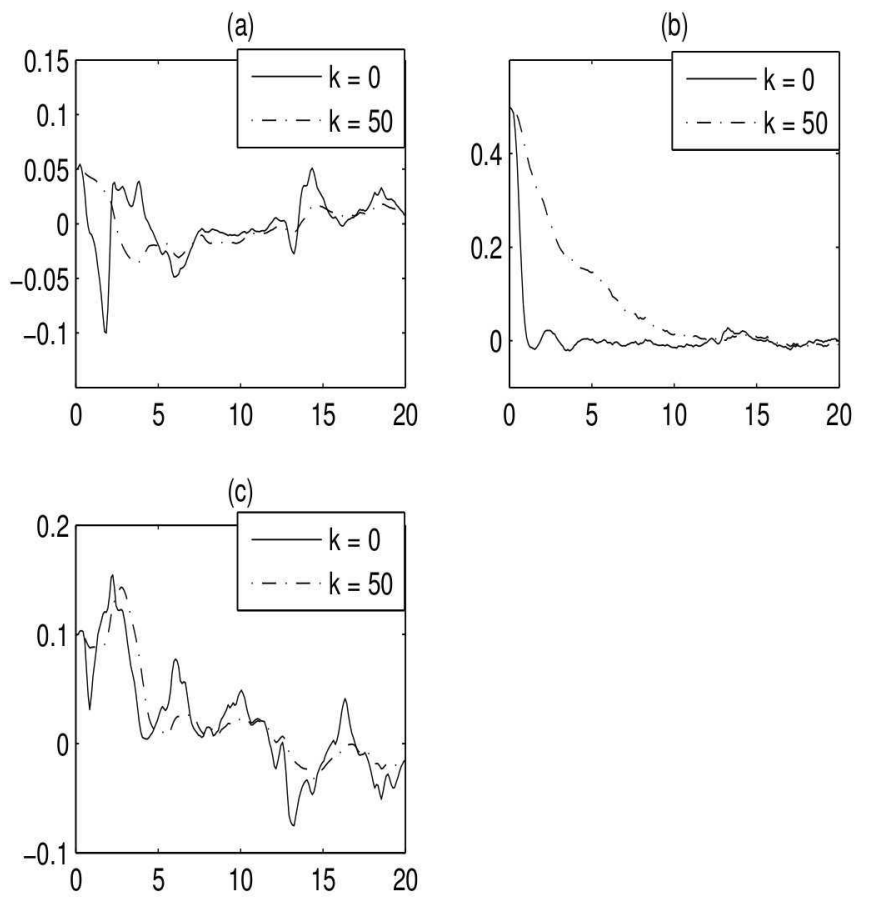

Fig. 5. Estimated values of u (a), v (b) and w (c) depending on $\mathrm{k}$
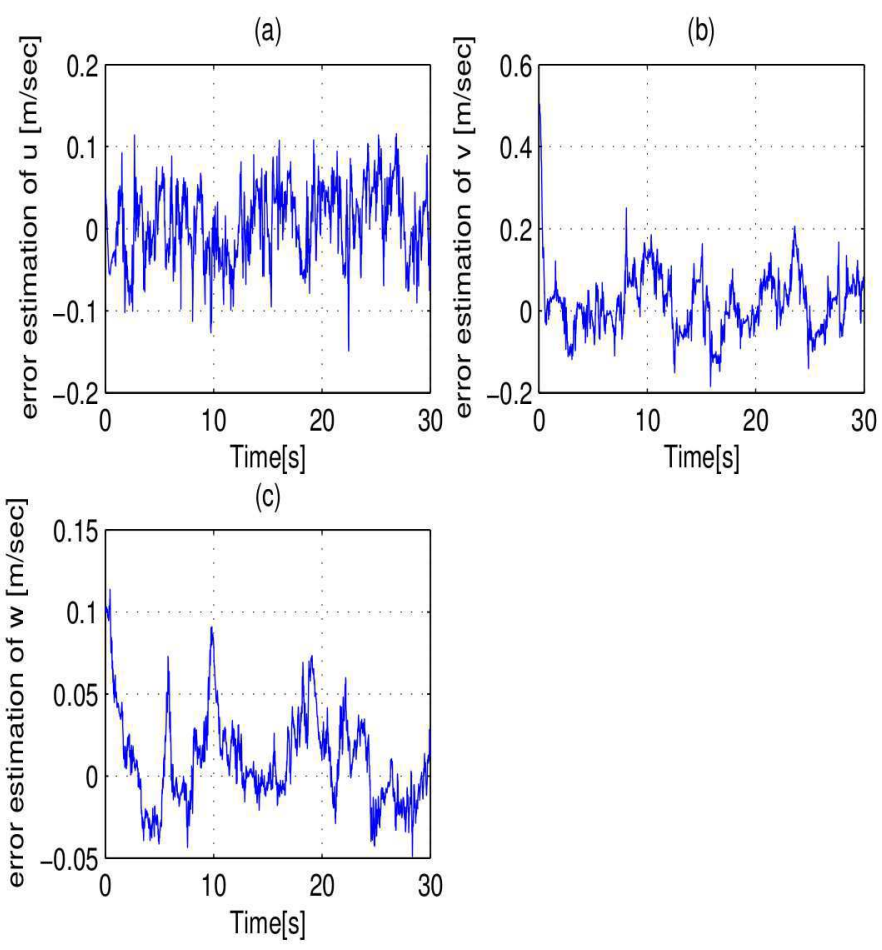

Fig. 6. Estimated values of u (a), v (b) and w (c) using the kalman filter 
Comparing fig. 5 and 6 , we can see that the estimation given by the adaptive observer is better than the Kalman filter estimation for the noisy acceleration considered in this section (see figure 3). The adaptive observer seems to be less sensitive to measurement noise than the Kalman filter. In fact, even when the value of the tuning parameter $k$ is equal to zero (no parameter to $k$ to guarantee the robustness to noise), the speed estimation is better than the one given by the Kalman filter.

\section{CONCLUSION}

In this paper, a solution to the classical problem of linear speed estimation has been presented when only linear acceleration and angular measurements are available. This corresponds to the case where no GPS is available in a UAV. A nonlinear robust adaptive observer, that guarantees arbitrary $L_{2}$ attenuation with respect to measurement noise, has been introduced This estimation strategy is compared to the classical Extended Kalman Filter approach. The theoretical results are confirmed via the simulations, in particular the noise attenuation by an arbitrary design parameter. An important issue concerns the persistence of excitation condition introduced by the adaptive algorithm that provides an observability condition to the linearized system. When this property is not satisfied, the linearized system becomes non-observable. The simulation results provide useful insight about the parameter tuning effect to achieve a satisfactory observer performance.

\section{REFERENCES}

Karl Astrom, Bjorn Wittenmark Computer Controlled Systems : Theory and Design. Prentice Hall, 1997.

N. Azouz, K. Benzemrane, G. Damm, G. Pradel. Modeling and development of a 4 rotors helicopter UAV. In Procedings of the 6th IFAC Symposium on Intelligent Autonomous Vehicles, Toulouse, September 3-5, 2007.

K. Benzemrane, G.L. Santosuosso, G. Damm. Unmanned aerial vehicle speed estimation via nonlinear adaptive observers. In Procedings of the 26th American Control Conference, New York, 11-13 July, 2007.

S. Bonnabel, P. Martin and P. Rouchon A non-linear symmetry-preserving observer for velocity-aided inertial navigation In Proceedings of the 2006 American Control Conference (ACC06), Minneapolis, Minnesota, USA, June 14-16, 2006.

P. Castillo, A. Dzul, R. Lozano. Real-time stabilization and tracking of a four-rotor mini rotorcraft. IEEE Transaction on control systems technology, vol. 12, pp. 510-516, July 2004.

G.R. Damm, R. Marino, F. Lamnabhi-Lagarrigue. Adaptive Nonlinear Output Feedback for Transient Stabilization and Voltage Regulation of Power Generators with Unknown Parameters. International Journal of Robust and Nonlinear Control, Vol. 14, pp 833-855, 2004.

N. Guenard, T. Hamel, R. Mahony. A practical Visual Servo Control for a Unmanned Aerial Vehicle In Proceedings of the 2007 IEEE International Conference on Robotics and Automation 10-14 April 2007.

N. Guenardt, T. Hamel, V. Moreaut. Dynamic modeling and intuitive control strategy for an "X4-flyer". International Conference on Control and Automation (ICCA2005), June 27-29, 2005, Budapest, Hungary MM-4.4
N. Makhloufi. Systeme Observateur pour un drone helicoptere. Laboratoire IBISC - CNRS / Universite d'Evry Val d'Essonne (in french). Juin 2007.

R. Marino, G. L. Santossuosso, P. Tomei. Robust Adaptive Observers for Nonlinear Systems with Bounded Disturbances. IEEE Transactions on automatic control, Vol. 46, No 6, pp 967-972, June 2001.

R. Marino, P. Tomei. Nonlinear Control Design - Geometric, Adaptive and Robust. Prentice Hall, Hemel Hempstead, London, 1995.

P. Pounds, R. Mahony, P. Hynes, J. Roberts. Design of a Four-Rotor Aerial Robot, In Procedings of 2002 Australasian Conference on Robotics and Automation, Auckland, 27-29 November 2002.

G. Pradel, K. Benzemrane, G. Damm, N. Azouz. Modeling and development of a quadrotor UAV. In Procedings of the 7th European Micro Air Vehicule Conference, Toulouse, September 17-21, 2007.

B. Vik and T. I. Fossen A nonlinear observer for GPS and INS integration. In Proceedings of the 40th IEEE Conference on Decision an Control, Orlando, Florida, USA, 2001

Y. Zhao, J. J. Slotine, Discrete nonlinear observers for inertial navigation. Systems and Control Letters, 2005. 